\title{
Modificación del proceso de reducción expansiva para la síntesis de nanopartículas de hierro
}

\author{
Adriana L. Rodríguez ${ }^{凶}$, Hugo R. Zea
}

\section{Changing the reductive expansion process to synthesize iron nanoparticles}

\begin{abstract}
The reductive expansion method has been used successfully in the synthesis of metal nanoparticles. However, there are no reports regarding the use of expansion methods to produce oxidized nanoparticles. Here, we propose an adjustment to the current oxidizing atmosphere application during reductive expansion. Mixtures of different weight ratios of iron nitrogen salts and urea (blowing agent) were subjected to thermal shock in an oven; the sudden sublimation of the urea and nitrogen salts originated a fine dispersion of material that came into contact with reducing gases. During this sublimation, metallic iron nanoparticles were produced and subsequently oxidized by the prevailing oxidizing atmosphere. Through characterization using X-ray diffraction, we determined that the crystalline phase of the formed nanoparticles depends on the composition of the reactants. In contrast, particle size distribution, surface area and oil absorption are not strongly influenced by this composition.
\end{abstract}

Keywords: Reductive expansion; iron oxide; nanoparticles; oxidation.

Edited by Beynor Paez $₫$ \& Alberto Acosta

Departamento de Ingeniería Química y Ambiental, Facultad de Ingeniería, Universidad Nacional de Colombia, Bogotá, Colombia.

Received: 22-11-2013 Accepted: 28-04-2014

Published on line: 09-05-2014

Citation: Rodríguez AL, Zea HR (2014) Modificación del proceso de reducción expansiva para la síntesis de nanopartículas de hierro. Universitas Scientiarum 19(2): 153-160 doi: 10.11144/Javeriana.SC19-2.mpre

Funding: NA

Electronic supplementary material: NA

\section{Introducción}

Los óxidos de hierro son uno de los compuestos más abundantes en la corteza terrestre y suelen encontrarse como $\mathrm{FeO}, \mathrm{Fe}_{2} \mathrm{O}_{3}$-hematita, $\gamma-\mathrm{Fe}_{2} \mathrm{O}_{3}$-maghemita, $\mathrm{Fe}_{3} \mathrm{O}_{4}$-magnetita o $\mathrm{FeO}(\mathrm{OH})$ - goethita (GollaSchindler et al. 2006). Se ha establecido que existe $\mathrm{Fe}_{2} \mathrm{O}_{3}$ amorfoycuatro polimorfos (alfa, beta, gamma yépsilon). Los tipos $\mathrm{Fe}_{2} \mathrm{O}_{3}$ gamma y épsilon son ferromagnético; el tipo alfa es llamado antoferromagnético mientras que el tipo beta es paramagnético (Chirita \& Grozescu 2009). La nanomagnetita $\mathrm{Fe}_{3} \mathrm{O}_{4}$ y la $\gamma-\mathrm{Fe}_{2} \mathrm{O}_{3}$ presentan excelentes propiedades magnéticas, las cuales les permiten ser usadas como ferritas blandas (Mohapatra \& Anand 2010) y para propósitos biomédicos debido a su biocompatibilidad (Cano et al. 2009) y 
biodegradabilidad (Unsoy et al. 2012). La hematita $\alpha-\mathrm{Fe}_{2} \mathrm{O}_{3}$ en un rango de tamaño de partícula nano, ha sido usada en la obtención de pinturas transparentes pues mejora su durabilidad, sombra, absorción UV y valor agregado; y dadas sus propiedades semiconductoras suele ser empleada en la conversión de la energía solar, fotocatálisis y en la disociación de agua (Chirita \& Grozescu 2009). De hecho, la fabricación de nanopartículas de hierro de valencias bajas resulta ser importante en el proceso de limpieza de aguas residuales (Nidhin et al. 2007).

El interés en la síntesis de óxidos de hierro ha crecido considerablemente debido al número de aplicaciones que poseen (Rahman et al. 2011). Son principalmente usados en construcción, pinturas y recubrimientos (Foley \& Wallace 2013), albañilería y baldosas; en la industria de plásticos, asfaltos, bitumen, barnices, pigmentos. Se han empleado en la preparación de catalizadores (Gerhard et al. 1999, Hutchings et al. 2006, Centomo et al. 2010, Pouran et al. 2014), adsorbentes, floculantes, sensores de gases, intercambiadores iónicos, en dispositivos magnéticos de grabación, dispositivos de almacenamiento de datos magnéticos(Fouda et al. 2012), tóneres y tintas para xerografía, en resonancia magnética, bioseparación (Cano et al. 2009, Niehaus et al. 2012) y medicina (Stroh et al. 2004).

El interés particular en su uso como material de construcción, se debe a su buena dispersabilidad y fuerza de teñido en baldosas, adoquines, cuadros, azulejos y concreto estampado. La industria de pinturas y recubrimientos, el segundo mayor consumidor de pigmentos de óxido de hierro, los emplea para lograr buenas dispersiones, obtener efectos óptimos en el color y mantener la resistencia en las películas de pintura, especialmente en aplicaciones de alto brillo y en la industria automotriz (Foley \& Wallace 2013). Además, las nanopartículas de óxido de hierro tienen buena capacidad para bloquear los rayos UV, lo que lo hace ideal en aplicaciones para el recubrimiento de vidrios.

Los nano óxidos de hierro han sido sintetizados por diferentes de métodos. Así, las partículas pueden ser producidas por métodos químicos húmedos, precipitación a temperatura ambiente (Mandel et al. 2011), mediación de surfactante, sol gel (Jitianu et al. 2002, Senoy et al. 2008), proceso hidrotérmico (Delgado et al. 2003), precipitación mediada con tensoactivo, descomposición térmica (Shao et al. 2005), emulsiónprecipitación, microemulsión precipitación (Chin \& Yaacob 2007), electro-deposición (Masanobu 2010), técnica hidrotérmica asistida por microondas, sonoquímica (Shafi et al. 2001, Srivastava et al. 2002), pirólisis por pulverización ultrasónica, métodos electroquímicos (Satyawati et al. 2006), entre muchos otros (Grabis et al. 2008, Teja \& Koh 2009, Mohapatra \& Anand 2010, Osipov et al. 2012).

Recientemente el método de síntesis de expansión reductiva (RES) fue introducido en la creación de nano y sub-micrométricas partículas de hierro y grafito recubierto de níquel; y aleaciones hierroníquel mediante calentamiento rápido de mezclas físicas de urea con un nitrato metálico (Luhrs et al. 2013). La técnica consiste en someter una mezcla de compuestos de nitrato y urea a rápido calentamiento a alta temperatura y bajo una atmosfera inerte, para crear nano y sub-micrométricas partículas de metales de valencia cero. Inicialmente el nitrato se descompone en óxido (en ausencia de urea) como es el caso de la síntesis por combustión; cuando las mezclas urea/ nitrato se someten a un calentamiento rápido hay una "convolución" de los procesos de descomposición. De esta forma, la urea se descompone generando gases reductores que permiten la formación del metal más que del óxido (Zea et al. 2011). Adicionalmente, la creación de partículas metálicas micro y submicrométricas a partir de precursores de óxido e hidróxido, indican que el uso de precursores óxidos para la completa reducción a micro-partículas, requiere mayor contenido de urea y mayores temperaturas; mientras que con el uso de hidróxidos se logra la reducción a temperaturas más bajas (Luhrs et al. 2013). Este hecho e investigaciones recientes, revelan que el método RES puede ser manipulado para crear un rango de micro y sub-micro partículas metálicas reducidas, apropiadas para diferentes aplicaciones (Leseman et al. 2011, Luhrs et al. 2013, Mowry et al. 2013).

\section{Materiales y Métodos}

Síntesis: El proceso desarrollado incluyó principalmente dos etapas: una mezcla física del nitrato de hierro con urea y un calentamiento rápido de dicha mezcla a $823 \mathrm{~K}$ bajo una atmósfera oxidante. 
Fe $\left(\mathrm{NO}_{3}\right)_{3} 9 \mathrm{H}_{2} \mathrm{O}$ (Nitrato de hierro nonahidratado, J.T. Baker Chemical Company 90-100\%) y $\mathrm{CH}_{4} \mathrm{NO}_{2}$ (Urea, Merck $\geq 90 \%$ en relaciones 1:1, 1:2, 2:1 en peso, fueron mezclados en un recipiente (mortero) hasta formar una pasta verde homogénea. Dicha mezcla fue transferida cuidadosamente a un bote de $4 \times 1 \times 1 \mathrm{~cm}$ procurando evitar la mayor cantidad de pérdidas.

Un tubo de alúmina de $2,54 \mathrm{~cm}$ de diámetro y $70 \mathrm{~cm}$ de longitud fue calentado en un horno tipo bisagra hasta alcanzar $823 \mathrm{~K}$. La temperatura se dejó estabilizar durante 120 segundos, momento el cual el bote fue introducido con ayuda de un alambre hasta el centro del tubo. El tiempo de reacción a dicha temperatura fue de 240 segundos para asegurar completa descomposición de la urea y el nitrato.

Durante el precalentamiento y la reacción, los extremos del tubo inmerso en el horno estuvieron expuestos al aire atmosférico permitiendo la entrada de oxígeno a lo largo del tubo y así mismo la formación de los óxidos de hierro; 190 segundos, luego de colocar el bote dentro el tubo de alúmina, en los extremos del tubo se observó la salida de un humo blanco por un tiempo aproximado de 15 segundos, momento en el cual se escuchó una leve explosión. El lapso transcurrido entre el momento de la introducción del bote y la salida del humo, representa el tiempo en que la urea presente en la mezcla alcanza su completa descomposición (Luhrs et al. 2013).

Elproceso fue simple y se garantizó un calentamiento rápido. Sin embargo, durante la reacción, la temperatura alcanzó a descender $\sim 5 \mathrm{~K}$ y se volvió a estabilizar $\sim 90$ segundos después. El horno se dejó enfriar hasta alcanzar la temperatura ambiente. Finalmente, el producto se retiró del bote y el material remanente en las paredes del tubo se removió con una espátula y se depositó en el recipiente de almacenamiento.

Caracterización: Muestras en polvo de los materiales sintetizados se analizaron por difracción de rayos $\mathrm{X}$ (DRX) en un difractómetro Scintag Pad V equipado con DataScan 3,1 software. Los resultados obtenidos se analizaron con el paquete computacional X'pert HighScore Plus utilizando la base de datos PDF-2 del ICDD. Para evaluar la estructura de superficie de la partícula y su morfología se uso microscopio de emisión de campo Zeiss Neon 40 y un microscopio electrónico de transmisión FEI tecnai-20; el pretratamiento para las muestras antes de ser evaluadas mediante microscopía electrónica consistió en disolver $2 \mathrm{mg}$ de material en solvente acuso con agitación ultrasónica por 30 minutos. El área superficial fue determinada por medio de la isoterma de adsorción-desorción de nitrógeno a $77 \mathrm{~K}$ ajustando los resultados por medio de modelo multicapas de la ecuación de BET. La distribución de tamaño de partículas fue determinada en un equipo de interferencia de rayo láser Malvern Zetasizer Nano $\mathrm{S}$ siguiendo el pretratamiento descrito para muestras caracterizadas por microscopía. El índice de absorción de aceite se determino siguiendo el procedimiento descrito en la norma ASTM D 234-82.

\section{Resultados}

Los resultados mostrados por DRX, indican la presencia de diferentes fases de óxido de hierro. (Figura 1). Para la mezcla 1:1 es posible identificar mayor proporción de $\mathrm{Fe}_{3} \mathrm{O}_{4}$, seguida por $\mathrm{Fe}_{2} \mathrm{O}_{3}$ y algo de $\mathrm{FeO}$; mientras que en la mezcla de relación 1:2 se observa principalmente la generación de $\mathrm{Fe}_{3} \mathrm{O}_{4}$ (Magnetita) con la aparición de wustita $(\mathrm{FeO})$ de acuerdo con lo mostrado por el pico $76.7^{\circ} 2 \theta$. En la mezcla 2:1 además de la presencia de $\mathrm{Fe}_{2} \mathrm{O}_{3}$ y $\mathrm{Fe}_{3} \mathrm{O}_{4}$ se observa la aparición de un nuevo pico en $42.0^{\circ} 2 \theta$, correspondiente a $\mathrm{Fe}_{2} \mathrm{O}_{3}$.

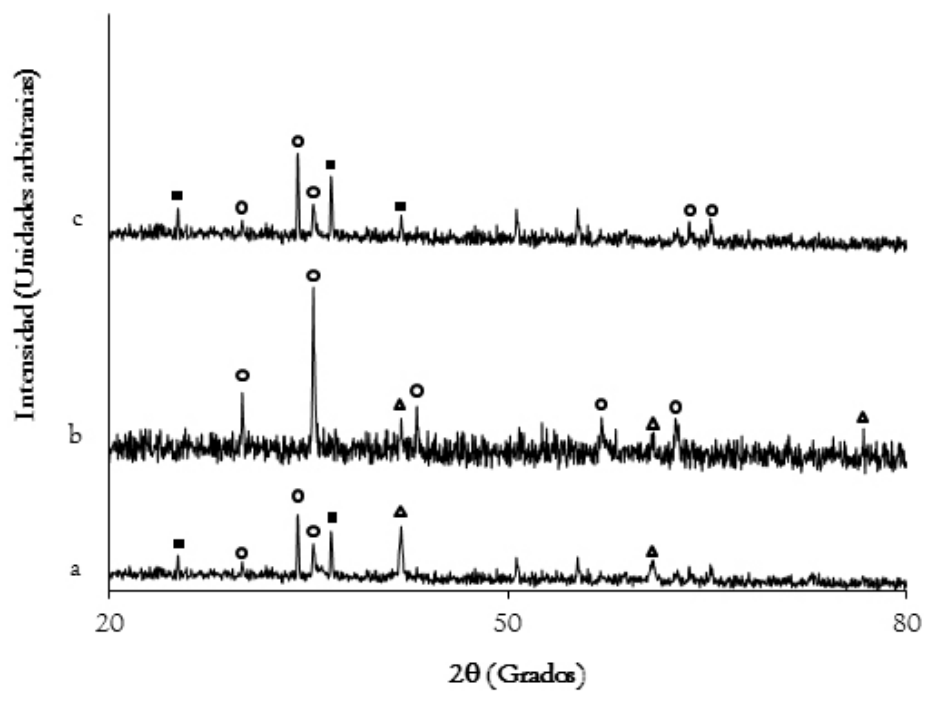

Fig. 1. Óxidos en DRX. a. Difractograma relación 1:1 donde se observa la presencia de tres fases $\mathrm{FeO}$ $(\Delta), \mathrm{Fe}_{2} \mathrm{O}_{3}(\mathbf{\square})$ y $\mathrm{Fe}_{3} \mathrm{O}_{4}(\mathbf{o})$. b. En la muestra relación 1:2 se evidencia únicamente la formación de $\mathrm{Fe}_{3} \mathrm{O}_{4}$ y FeO. c. Muestra 2:1, donde se aprecia la presencia de $\mathrm{Fe}_{3} \mathrm{O}_{4}$ y $\mathrm{Fe}_{2} \mathrm{O}_{3}$. 
Para cada una de las curvas de distribución de tamaño de partícula (Figura 2), es posible determinar un diámetro de partícula promedio de 182, 192 y $154 \mathrm{~nm}$ en las muestras 1:1,1:2 y 2:1, respectivamente, a partir de los valores extremos de cada curva. Esto permite concluir que el tamaño de la mayor parte de las partículas obtenidas se encuentra entre los 154 y $192 \mathrm{~nm}$. En la muestra de proporciones iguales de urea y nitrato, el rango resulta ser un poco más amplio comparado con los resultados obtenidos en las relaciones $1: 2$ y $2: 1$.

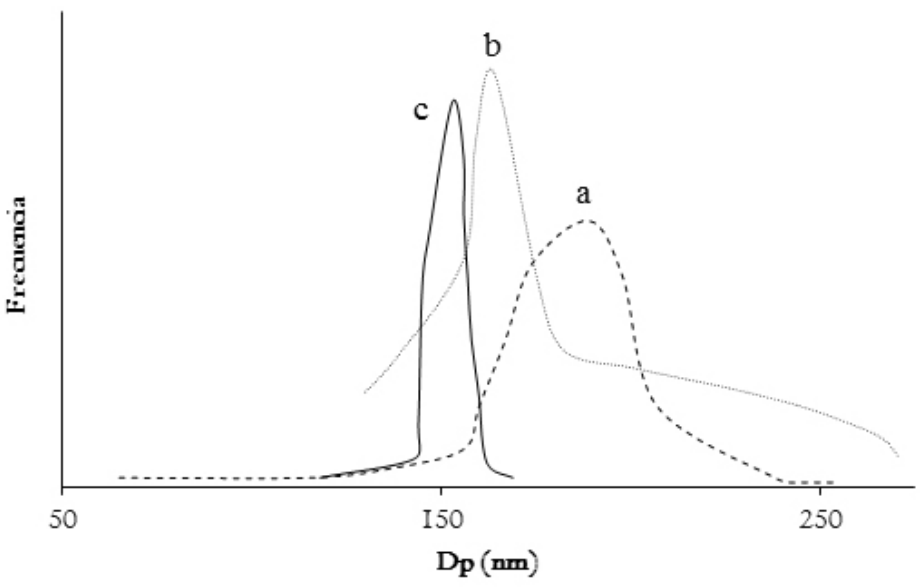

Fig. 2. Distribución de tamaño de partícula de óxido de hierro para las muestras preparadas relación en peso Fe $\left(\mathrm{NO}_{3}\right)_{3}: \mathrm{CH}_{4} \mathrm{NO}_{2}$ a. 1:1, b. 1:2, c. 2:1.

Las imágenes de SEM (Figura 3a) y TEM (Figura 3b) corresponden a la muestra 1:2. La fotografía de SEM revela aglomerados micrométricos compuestos

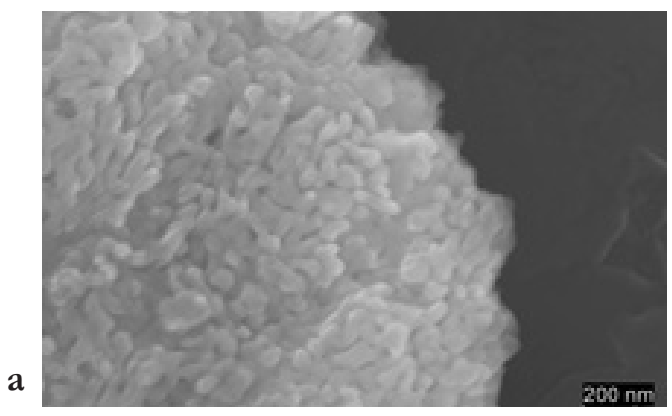

de partículas del orden de 150-200 nm, mientras que la fotografía de TEM permite diferenciar la formación de partículas más pequeñas de hasta 10 y $20 \mathrm{~nm}$. Estos resultados son congruentes con lo reportado en el análisis de diámetro de partícula, y son consecuencia de la disgregación de las partículas aglomeradas debido al proceso de agitación ultrasónica.

Los resultados arrojados por el análisis BET revelan áreas superficiales muy cercanas entre sí, 45, 51 y $54 \mathrm{~m}^{2} / \mathrm{g}$ para las muestras 1:1,1:2, 2:1 respectivamente; con variaciones no mayores a $10 \mathrm{~m}^{2}$.

La prueba de absorción de aceite parece no tener cambios: 34,35 y $35 \mathrm{~cm}^{3} / \mathrm{g}$, para las muestras 1:1, 1:2, 2:1 respectivamente; hecho que concuerda con la pequeña variación en el área superficial anteriormente mencionada.

\section{Discusión}

Estudios anteriores demostraron que la técnica denominada síntesis de reducción expansiva (RES por su sigla en inglés Reduction Expansive Synthesis) genera aglomerados micrométricos fácilmente disgregables de nanopartículas metálicas(Zea et al. 2011, Luhrs et al. 2013). Estos resultados mostraron que partículas cero valentes se pueden producir a partir de sales nitrogenadas del metal de interés, por medio de la descomposición súbita de urea. La urea se descompone en dos etapas bien diferenciadas: la primera etapa sucede en el intervalo de temperatura de 493-523 K, y luego una segunda etapa entre 633 y $653 \mathrm{~K}$. En la primera etapa se ha postulado la descomposición de la urea para formar amoniaco y ácido isociánico:

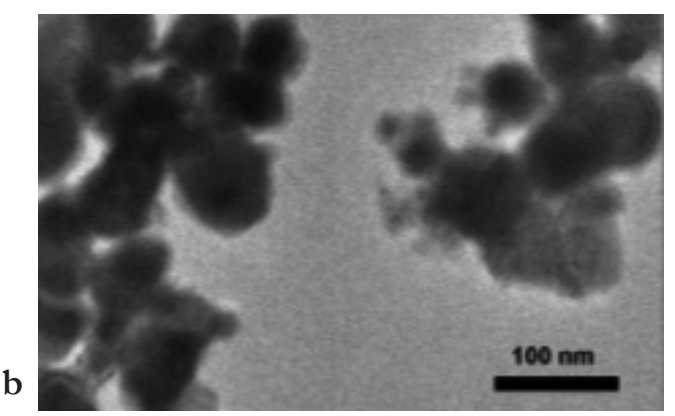

Fig. 3. Microfotográficas electrónicas de la muestra 1:2. a. Microfotográfica electrónica de barrido. b. Microfotográfica electrónica de transmisión. 


$$
\left(\mathrm{NH}_{2}\right)_{2} \mathrm{CO} \rightarrow \mathrm{NH}_{3}+\mathrm{HNCO}
$$

La segunda etapa se representará principalmente amoníaco y dióxido de carbono

$$
\mathrm{HNCO}+\mathrm{H}_{2} \mathrm{O} \rightarrow \mathrm{NH}_{3}+\mathrm{CO}_{2}
$$

Dicha descomposición resulta ser muy dependiente de la composición inicial de la mezcla reaccionante (Zea et al. 2011). Por ejemplo, ha sido reportado que en presencia de agua, productos como el ácido ciánico, ácido cianúrico y algunas moléculas de mayor peso molecular son generadas, todos ellos con una tendencia químico reductora.

A diferencia del método RES anteriormente mencionado, con la modificación realizada se busca la formación de óxidos en lugar de la obtención de partículas cero valentes a partir de las mismas fuentes. De esta forma la modificación se origina cambiando parte de la atmosfera de reacción, manteniendo la atmósfera reductora, pero exponiéndola durante todo el proceso al aire atmosférico y por tanto a oxígeno, el cual se encarga la oxidación. Dependiendo la cantidad de oxígeno que pueda reaccionar con las partículas metálicas y la cantidad de gases reductores se obtendrán óxidos de hierro con diferentes estados de oxidación.

Así el hierro metálico formado a partir de RES reacciona con el oxígeno para formar $\mathrm{FeO}$

$$
\mathrm{Fe}+1 / 2 \mathrm{O}_{2} \rightarrow \mathrm{FeO}
$$

Este a su vez en presencia de oxigeno en la atmósfera puede formar $\mathrm{Fe}_{3} \mathrm{O}_{4}$.

$$
3 \mathrm{FeO}+1 / 2 \mathrm{O}_{2} \rightarrow \mathrm{Fe}_{3} \mathrm{O}_{4}
$$

Cuando las condiciones de reacción favorecen la oxidación del $\mathrm{FeO}$ (ausencia de una atmósfera reductora), el producto será $\mathrm{Fe}_{2} \mathrm{O}_{3}$.

$$
4 \mathrm{Fe}_{3} \mathrm{O}_{4}+\mathrm{O}_{2} \rightarrow 6 \mathrm{Fe}_{2} \mathrm{O}_{3}
$$

De acuerdo con los picos encontrados en los difractogramas (Figura 1), las fases presentes se ven afectadas por la relación entre la urea y el nitrato. Cuando la relación es 1:1 se encuentran tres fases $\mathrm{Fe}_{2} \mathrm{O}_{3}$, $\mathrm{Fe}_{3} \mathrm{O}_{4}$ y $\mathrm{FeO}$ (con $\mathrm{Fe}_{3} \mathrm{O}_{4}$ en mayor proporción). En el momento que la cantidad de nitrato es incrementada, el FeO desaparece y se revelan picos correspondientes a $\mathrm{Fe}_{3} \mathrm{O}_{4}$ y $\mathrm{Fe}_{2} \mathrm{O}_{3}$ únicamente. En este caso, la cantidad de oxígeno presente es capaz de llevar parte del hierro a su mayor estado de oxidación. En la relación 1:2 (mayor contenido de urea) se observa un aumento significativo en la intensidad de los picos de $\mathrm{Fe}_{3} \mathrm{O}_{4}$ y la ausencia del $\mathrm{Fe}_{2} \mathrm{O}_{3}$. La no formación de $\mathrm{Fe}_{2} \mathrm{O}_{3}$ es debida a la presencia de mayor cantidad de gases reductores en la atmósfera, provenientes de la descomposición de la urea, lo que dificulta el paso de oxígeno e impide la completa oxidación del hierro. Claramente el hierro metálico no puede mantenerse en valencia cero, como en el caso de la RES convencional, pues la presencia de oxígeno en la atmosfera reaccionante necesariamente oxida el hierro, formando así los óxidos presentes en cada una de las muestras.

Por otro lado, el diámetro promedio de partícula (Figura 2) parece no verse afectado significativamente por el método de preparación. Aunque el tamaño promedio difiera en los tres casos, la dispersión observada entre las muestras no supera los $38 \mathrm{~nm}$.

El área superficial determinada por el método BET entre una y otra muestra resulta ser pequeña, prácticamente no varía. Este hecho se ve reflejado también en la prueba de absorción de aceite, lo que implica que independientemente de la forma de preparación de cada una de las muestras, las propiedades de tamaño, área y capacidad de absorción de aceite no se ven fuertemente influenciadas por la presencia de mayor proporción (bien sea de urea o nitrato) durante el proceso de preparación.

Comparando el material resultante de las tres preparaciones con marcas comerciales como Polysciences, Inc., Cathay Pigments Ltda., Yipin Pigments, Inc., las muestras preparadas con RES modificado resultan tener un diámetro promedio muy cercano a las características de los pigmentos ofrecidos por dichas compañías (200 nm). Sin embargo, difieren en el área superficial en aproximadamente 20 - $30 \mathrm{~m}^{2} / \mathrm{g}$ y en la capacidad de absorción de aceite en $10-20 \mathrm{~g} / 100 \mathrm{~g}$, parámetros de gran interés en la formulación de pinturas.

ElRES modificado demostró ser un procedimiento apropiado para obtener nanopartículas oxidadas de tamaños cercanos a los $200 \mathrm{~nm}$. Solo se consideró el efecto de la variación en la relación urea-nitrato, por 
lo que sería interesante evaluar los efectos que pueden tener otras variables de proceso como temperatura y tiempo en la distribución del tamaño de partícula y la fase predominante en el material sintetizado; además la facilidad y sencillez del procedimiento desarrollado muestra que puede ser un proceso escalable y eficiente para la síntesis de nanopartículas.

\section{Conclusión}

Partículas de tamaños nanométricos fueron obtenidos a partir del método RES modificado. Los resultados de caracterización permiten concluir que la fase cristalográfica obtenida es dependiente del método de preparación, más exactamente de la relación en peso de los reactivos. Las propiedades determinadas como tamaño de partícula, porosidad, área superficial, y capacidad de absorción de aceite, resultan ser independientes de la relación en peso usada durante la síntesis.

\section{Agradecimientos}

Los autores expresan su gratitud al laboratorio de Catálisis del Laboratorio de Ingeniería Química de la Universidad Nacional de Colombia y a la Prof. Claudia C. Luhrs, Mechanical and Aerospace Engenieering Department, Naval Postgraduate School, Monterey, CA.

\section{Conflicto de Intereses}

Los autores declaran que en el desarrollo de los experimentos descritos y el análisis de los resultados presentados en este artículo, no existe conflicto de intereses.

\section{Referencias}

Cano LA, Cagnoli MV, Stewart SJ, Cabanillas ED, Romero EL, Marchetti SG (2009) Synthesis and characterization of superparamagnetic iron oxide nanoparticles for biomedical applications. En: Desimoni J, Ramos CP, Arcondo B, Saccone FD, Mercader RC (ed) LACAME 2008. Springer Berlin Heidelberg, pp 275-280

Centomo P, Zecca M, Di Noto V, Lavina S, Bombi GG, et al. (2010) Characterization of Synthetic Iron Oxides and their Performance as Support for $\mathrm{Au}$ Catalysts. ChemCatChem 2(9):1143-1149 doi 10.1002/ cctc.201000049

Chin AB, Yaacob II (2007) Synthesis and characterization of magnetic iron oxide nanoparticles via w/o microemulsion and Massart's procedure. Journal of Materials Processing Technology 191(1-3):235-237 doi http://dx.doi.org/10.1016/j.jmatprotec.2007.03.011
Chirita M, Grozescu I (2009) $\mathrm{Fe}_{2} \mathrm{O}_{3}$ - Nanoparticles, Physical Properties and their photochemical and photoelectrochemical applications. Buletinul SStiintific al Universitătuii "Politehnica" din Timisoara ROMÂNIA 54:1

Delgado E, Bohórquez A, Pérez AlcázarG, Bolaños A (2003) Study of the Evolution in the Iron Oxide Synthesis by Hydrothermal Process. Hyperfine Interactions 148-149(14):129-134 doi 10.1023/B:HYPE.0000003773.12577.7f

Foley R, Wallace T (2013) Iron Oxide Nanoparticles as Additives in Construction Materials and Industrial Coatings. Consultado September

Fouda MFR, El-Kholy MB, Moustafa SA, Hussien AI, Wahba MA, El-Shahat MF (2012) Synthesis and Characterization of Nanosized $\mathrm{Fe}_{2} \mathrm{O}_{3}$ Pigments. International Journal of Inorganic Chemistry 2012:9 doi 10.1155/2012/989281

Gerhard EE, Helmut KE, Ferdi SE, Jens WE (1999) Handbook of Heterogeneous Catalysis

Golla-Schindler U, Hinrichs R, Bomati-Miguel O, Putnis A (2006) Determination of the oxidation state for iron oxide minerals by energy-filtering TEM. Micron 37(5):473-477 doi http://dx.doi.org/10.1016/j.micron.2005.11.002

Grabis J, Heidemane G, Rasmane D (2008) Preapartion of $\mathrm{Fe}_{3} \mathrm{O}_{4}$ and $\mathrm{Fe}_{2} \mathrm{O}_{3}$ Nanoparticles by Liquid and Gas Phase Process. Materials Science (MEDŽIAGOTYRA) 14(4):292-295

Hutchings GJ, Hall MS, Carley AF, Landon P, Solsona BE, Kiely CJ,et al. (2006) Role of gold cations in the oxidation of carbon monoxide catalyzed by iron oxidesupported gold. Journal of Catalysis 242(1):71-81 doi http://dx.doi.org/10.1016/j.jcat.2006.06.001

Jitianu A, Crisan M, Meghea A, Rau I, Zaharescu M (2002) Influence of the silica based matrix on the formation of iron oxide nanoparticles in the $\mathrm{Fe}_{2} \mathrm{O}_{3}-\mathrm{SiO} 2$ system, obtained by sol-gel method. Journal of Materials Chemistry 12(5):1401-1407 doi 10.1039/B110652J

Leseman Z, Luhrs C, Phillips J, Soliman H (2011) Aerosol Reduction/Expansion Synthesis (A-RES) for zero valent metal particles

Luhrs C, Kane M, Leseman Z, Phillips J (2013) Novel Process for Solid State Reduction of Metal Oxides and Hydroxides. Metallurgical and Materials Transactions B 44(1):115-122

Mandel K, Hutter F, Gellermann C, Sextl G (2011) Synthesis and stabilisation of superparamagnetic iron oxide nanoparticle dispersions. Colloids and Surfaces $A$ : Physicochemical and Engineering Aspects 390(1-3):173-178 doi http://dx.doi.org/10.1016/j.colsurfa.2011.09.024

Masanobu I (2010) Electrodeposition of Iron and Alloys. En: Paunovic MSaM (ed) Modern Electroplating. John Wiley \& Sons, Inc., pp 317-318 
Mohapatra M, Anand S (2010) Synthesis and applications of nano-structured iron oxides/hydroxides - a review. International Journal of Engineering, Science and Technology 2(8):127-146

Mowry M, Palaniuk D, Luhrs CC, Osswald S (2013) In situ Raman spectroscopy and thermal analysis of the formation of nitrogen-doped graphene from urea and graphite oxide. RSC Advances doi 10.1039/ C3RA42725K

Nidhin M, Indumathy R, Sreeram KJ, Nair BU (2007) Synthesis of iron oxide nanoparticles of narrow size distribution on polysaccharide templates. Bulletin of Materials Science - Springer 31(1):93-96

Niehaus J, Becker S, Schmidtke C, Werner K, Weller H (2012) Precision Synthesis of Iron Oxide Nanoparticles and Their Use as Contrast Agents. En: Buzug TM, Borgert J (ed) Magnetic Particle Imaging. Springer Berlin Heidelberg, pp 87-91

Osipov VV, Platonov VV, Uimin MA, Podkin AV (2012) Laser synthesis of magnetic iron oxide nanopowders. Tech Phys 57(4):543-549 doi 10.1134/ S1063784212040214

Pouran SR, Raman AA, Wan Daud WMA (2014) Review on the application of modified iron oxides as heterogeneous catalysts in Fenton reactions. Journal of Cleaner Production 64(0):24-35 doi http://dx.doi. org/10.1016/j.jclepro.2013.09.013

Rahman MM, Khan SB, Jamal A, Faisal M, Aisiri AM (2011) Iron Oxide Nanoparticles Nanomaterials. www.intechopen.com, http://cdn.intechopen.com/ pdfs/25341/InTech-Iron_oxide_nanoparticles.pdf, pp 43-66

Satyawati JS, Prajakta PR, Madhav N, P PB (2006) Role of ligands in the formation, phase stabilization, structural and magnetic properties of $\alpha-\mathrm{Fe}_{2} \mathrm{O}_{3}$ nanoparticles. $J$ Nanopart Res 8(5):635-643 doi 10.1007/s11051-0059033-x

Senoy T, D S, Yasuhiko Y, M RA (2008) Spectroscopic and photoluminescence studies on optically transparent magnetic nanocomposites based on sol-gel glass: Fe3O4. J Nanopart Res 10(1):203-206 doi 10.1007/ s11051-007-9217-7

Shafi KVPM, Ulman A, Yan X, Yang NL, Estournès C, White H, Rafailovich M (2001) Sonochemical Synthesis of Functionalized Amorphous Iron Oxide Nanoparticles. Langmuir 17(16):5093-5097 doi 10.1021/ la010421+

Shao H, Lee H, Huang Y, InYong K, Kim C (2005) Control of iron nanoparticles size and shape by thermal decomposition method. Magnetics, IEEE Transactions on 41(10):3388-3390 doi 10.1109/ TMAG.2005.855206
Srivastava DN, Perkas N, Gedanken A, Felner I (2002) Sonochemical Synthesis of Mesoporous Iron Oxide and Accounts of Its Magnetic and Catalytic Properties. The Journal of Physical Chemistry B 106(8):1878-1883 doi 10.1021/jp015532w

Stroh A, Zimmer C, Gutzeit C, Jakstadt M, Marschinke F, et al. (2004) Iron oxide particles for molecular magnetic resonance imaging cause transient oxidative stress in rat macrophages. Free Radical Biology and Medicine 36(8):976-984 doi http://dx.doi.org/10.1016/j. freeradbiomed.2004.01.016

Teja AS, Koh PY (2009) Synthesis, properties, and applications of magnetic iron oxide nanoparticles. Progress in Crystal Growth and Characterization of Materials 55(1-2):22-45 doi http://dx.doi.org/10.1016/j. pcrysgrow.2008.08.003

Unsoy G, Yalcin S, Khodadust R, Gunduz G, Gunduz U (2012) Synthesis optimization and characterization of chitosan-coated iron oxide nanoparticles produced for biomedical applications. J Nanopart Res 14(11):1-13 doi 10.1007/s11051-012-0964-8

Zea H, Luhrs CC, Phillips J (2011) Reductive/expansion synthesis of zero valent submicron and nanometal particles. Journal of Materials Research 26(05):672-681 
Modificación del proceso de reducción expansiva para la síntesis de nanopartículas de hierro

Resumen. La metodología de expansión reductiva ha sido exitosa en la síntesis de nanopartículas metálicas. Sin embargo, no hay reportes en la literatura respecto al uso de métodos de expansión para la producción de nanopartículas oxidadas. Se propuso una modificación en la aplicación de corriente de atmósfera oxidante durante el proceso de expansión reductiva. Mezclas de diferentes relaciones en peso de sales nitrogenadas de hierro y urea (agente expansor), fueron sometidas a choque térmico en un horno donde la sublimación súbita de la urea y las sales nitrogenadas, generaron una dispersión fina de material que entró en contacto con gases reductores. Durante esta sublimación se propició la formación de nanopartículas metálicas de hierro subsecuentemente oxidadas por la atmósfera oxidante reinante. Caracterización por medio de difracción de rayos $\mathrm{X}$ permitió concluir que el tipo de fase cristalina de las nanopartículas formadas es dependiente de la composición de los reactantes; por el contrario, la distribución de tamaño de partícula, área superficial y absorción de aceite no se ve fuertemente influenciados por dicha composición.

Palabras clave: Expansión reductiva; óxido de hierro; nanopartículas; oxidación.
Modificaçáo do processo de reduçáo expansiva para a síntese de nanopartículas de ferro

Resumo. A metodologia da expansão redutiva tem sido exitosa na síntese de nanopartículas metálicas. No entanto, não tem sido reportado na literatura respeito ao uso de métodos de expansão para a produção de nanopartículas oxidadas. Foi proposta uma modificação na aplicação da corrente de atmosfera oxidante durante o processo de expansão redutora. Mistura de diferentes relações em peso de sais nitrogenados de ferro e ureia (agente expansor), foram submetidas a choque térmico num forno onde a sublimação súbita da ureia e os sais nitrogenadas, geraram uma dispersão fina de material que entrou em contacto com gases redutores. Durante esta sublimação proporcionou-se a formação de nanopartículas metálicas de ferro subsequentemente oxidadas pela atmosfera oxidante reinante. Caracterização por meio de difração de raios-X permitiu concluir que o tipo de fase cristalina das nanoparticulas formadas é dependente da composição dos reagentes; pelo contrario, a distribuição do tamanho da partícula, área superficial e absorção de óleo não se vêm fortemente influenciados por dita composição.

Palavras-chave: Expansão redutora; óxido de ferro; nanopartículas; oxidação. 